\title{
Recurrent Backpropagation Networks receiver for modulated signals over noisy channels
}

\author{
A. Canegalli*, L. Favalli*, A. Mecocci**, R. Pizzi* \\ * University of Pavia \\ via Ferrata, 1, \\ I-27100 Pavia, Italy \\ tel: $+39(0) 382505923$ \\ fax: $+39(0) 38242258$ \\ e-mail:lorenzo@comell.unipv.it \\ ** University of Siena \\ via Roma,77, \\ I-53100 Siena, Italy \\ tel: $+39(0) 577263604$ \\ fax: $+39(0) 577263602$
}

\begin{abstract}
Recurrent Backpropagation networks have been used to build up a neural receiver for GSM signals. The simulations have been carried out considering an AWGN channel, corrupted by ISI, fading and Doppler. The experimental results show that the neural receiver performs better than a classic choerent one and it improves its performances when the number of training samples is increased.
\end{abstract}

\section{INTRODUCTION}

The growing spread of mobile radio systems and their severe channel constraints: [1] are leading to the search for new techniques which could reconstruct a satisfactory signal using methods ccmputationally less expensive than Viterbi equalizers and both coherent and noncoherent demodulators.

As equalization can be seen as an inverse filtering problem [2] and demodulction is substantially a mapping of received signals on an expected set of symbols, Neural Networks can put themselves as efficient alternatives to the classical techniques because it is well-known that they behave as nonlinear filters [3] and are powerful classification tools [4]. Besides, the traditional approach uses linear filtering techniques, while the channel has non-linear features and Neural Networks show interesting performances in treating non-linearity [5]

For these reasons we wished to experiment a fully neural receiver (equalizer plus demodulator). In order to cope with the time-varying features of the channel, we chose to adopt Recurrent Networks, which have shown [6] to be the most powerful neural architecture in modeling and forecasting the behavior of dynamic systems. In the following section we will justify this assumption. The other sections are devoted to the description of our model and to the experimental results.

\section{DYNAMICAL NETTWORKS FOR SPATIO TEMPORAL. PATTERNS}

We can define as spatio-temporal pattern a function $x(t)$ which maps any time $t$ into one point in the $n$ dimensional input space: $x(t):\left[t_{0}, t_{1}\right] \rightarrow R^{n}$
It is possible to represent a spatio-temporal pattern as a trajectory $x(t)$ in the input space, and aim of the neural network is to implement a transformation that associates to the function $x(t)$ an output function $y(t)$ at any time $t$. Several methods have been proposed in the past for spatio-icmporal neural processing [7]. The eldest idea has been that of transforming temporal data into a sequence of data. When a new input set is received, the previous data are eliminated, and so on. Afterwards timedelay networks have been studied, where the information at the time $t$ is shifted in a chain of nodes: the number of nodes determines the number of time intervals on which the information is sampled. Besides, neural networks that accept frequency-encoded information have been proposed, actually adopting the method of choice of biological sensors. Another method consists of modifying the neurons in such a way that they sum up the data over time, allowing a slow decay of historical information.

Most of the networks above described are actually networks without memory. Better performances have been provided by strictly dynamical networks whose architecture is characterized by a state feedback [5] realized through suitable connections among nodes. In the state feedback the nodes receive as incoming signal both the network inputs and the output of the other nodes including its own.

A network frequently used as a dynamical model is the Multilayer Perceptron (MLP). Actually, this network model is still a static one, which processes time series data by converting the temporal sequence into a static pattern by unfolding the sequence over time, but it is possible to give the MLP a recurrent architecture. Introduced by Rumelhart and Williams [8] and improved by Pineda [9], this model, called backpropagation recurrent network (RBP) presents input vectors $v_{1}, \ldots, v_{n}$, and output vectors $\mathrm{u}_{1}^{\prime}, \ldots, \mathrm{u}_{\mathrm{N}}^{\prime} \ldots$

The output from the first layer is

$z_{j}=\left\{\begin{array}{cc}u_{j}(k) & 0 \leq j \leq N \\ v_{j}(k) & N+1 \leq j \leq L\end{array}\right.$

0-7803-31-09-5/96/\$5.00 C 1996 IEEE 
where $\mathrm{z}_{\mathrm{j}}$ is the output from the first layer, $\mathrm{v}_{\mathrm{j}}$ is the current input and $z_{0}$ is the possible bias node.

The current output is:

$\mathrm{u}_{\mathrm{i}}^{\prime}(\mathrm{k}+1)=\mathrm{f}\left(\mathrm{I}_{\mathrm{i}}(\mathrm{k})\right)$

where

$I_{i}(k)=\sum_{j=1}^{L} w_{i j} z_{j}(k)+z_{0}$

Thus the node equation [5] is

$\mathrm{u}(\mathrm{k}+1)_{\mathrm{i}}=\mathrm{f}\left(\sum_{\mathrm{j}}^{\mathrm{n}} \mathrm{w}_{\mathrm{ij}} \mathrm{u}_{\mathrm{j}}(\mathrm{k})+\mathrm{v}_{\mathrm{j}}(\mathrm{k})\right)$

where $\mathrm{f}$ is the sigmoid function .

The learning algorithm of the RBP network is similar to that of the static MLP,. As for the standard backpropagation, this algorithm is based on the search for the minimum error $F(W)$ using the descent gradient technique

\section{THE NEURAL RECEIVER}

The Neural Receiver (NR) presented in this paper has been realized using RBP networks. The NR has been tested using QPSK modulation with a 6 poles Butterworth filter with bandwith $.75 / \mathrm{Tb}$ limiting the bandwidth of a fading channel with Doppler effect.

- Information is coded by means of a NRZ sequence divided into packets using the same structure as the GSM burst [1] (Fig. 1)..

The base band representation of a QPSK modulated signal is made up of two quadrature that have been processed simultaneously by the same network. The

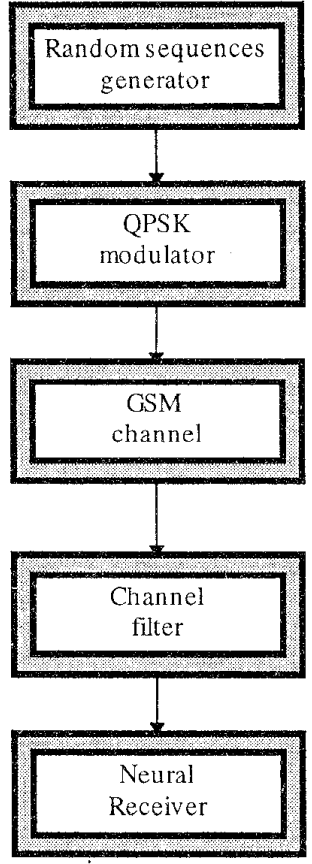

Fig. 1 - The employed GSM communication system.

number of nodes of the RBP network employed in the experiments for QPSK demodulation has a been selected experimentally, in our case, with QPSK signals, best performances have been obtained using a network with 74 input nodes, 148 hidden nodes and 74 output nodes, which allows the network to maintain a simmetry with respect to the middle part of the QPSK burst, that is a known sequence of bits.. The network has been trained with noise corrupted symbols.

observations of the input signal are provided through a delay line whose number of taps has been set equal to the number of input nodes. The equalizer produces an output which is a function of both the previous outputs and the inputs on the delay line. (Fig. 2).

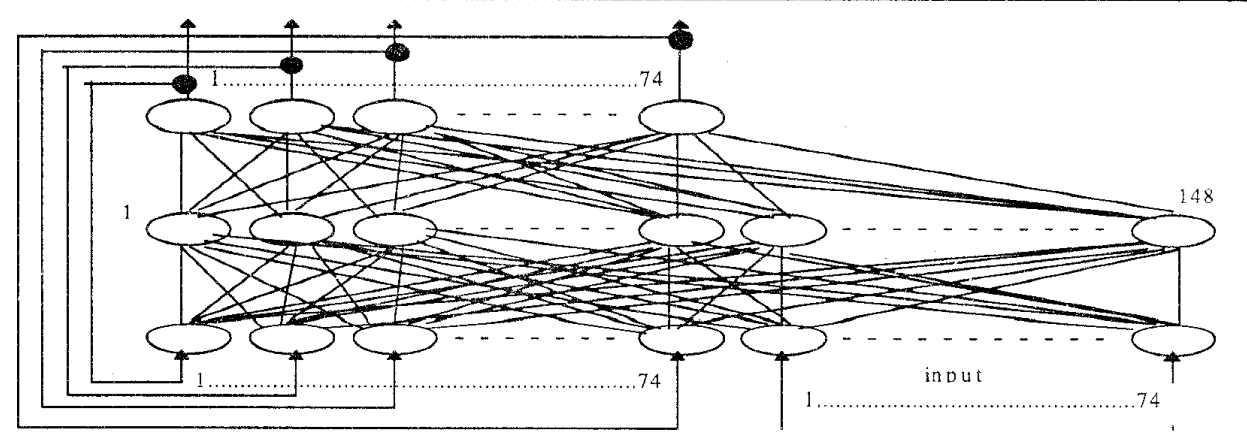

Fig 2 - Recurrent Backpropagation Network used. 


\section{IV.EXPERIMENTAL RESULTS}

The performances of the reural receiver have been tested under different working conditions and compared with those of a coherent system to account for both transmitter, receiver and channel band limits.

In particular, we have used a Butterworth filter in order to obtain a better simulation of the real transmission conditions. Besides, the filtering has introduced a high grade of intersymbol interference (ISI) that has helped the network task by increasing the correlation between the received symbols.

Symulation programs have been written in $\mathrm{C}$ on DecStation 5000/240. On such a platform, the time necessary to achieve corivergence, with a MSE lower than 0.001 on the given training set has been always less than 24 hours.

The results obtained during the testing have shown a good performance of the network with the growing of the sample. In fact the network has always come to convergence, even though the time required becomes longer and longer while the number of samples is increased and the channel parameters are set to simulate worse transmission conditions. However, during the testing phase, the network has shown to improve its performances with the growing of the learnt sample (Fig. 3)

In the following we report the graphic showing the generalization capabilities of the network with respect to the bit number used for the training (AWGN channel with ISI, fig. 4).

The subsequent graphics (Fig. 5, Fig. 6, Fig. 7 ) show the behaviour of the BER with the growing of the SNR for a neural receiver trained with 7104 samples, in the channel conditions typical of GSM systems simulations with gaussian noise, fading, Doppler effect in three environmebts:

- rural area

- hilly terrain

- urban area

\section{CONCLUSIONS AND FUTURE RESEARCHES}

The work has shown the plausibility of a neural receiver able to recover a GSM signal affected by gaussian noisc, ISI, fading, Doppler effect on any terrain.

An advantage of such a tool is its virtual independency from the SNR variations.

Our experiments indicated that, having the possibility of training the network with a high number of samples (using parallel computers or very long times on standard workstations), it will be possible to highly improve the network performances, currently already better than those of the c.assical choerent receiver even in prohibitive conditions.

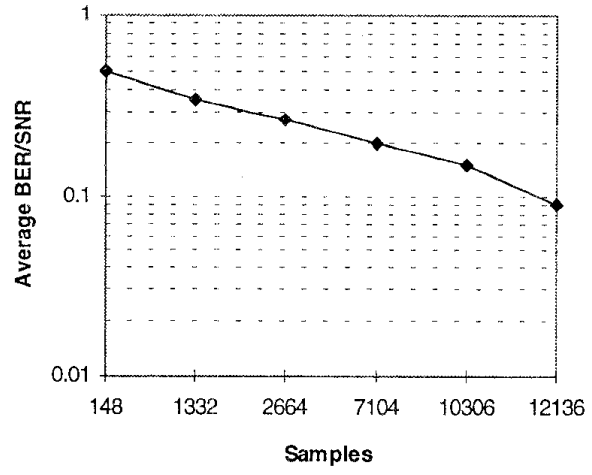

Fig. 3 - Performances improvement of the network depending on the sample number.



Fig. 4 - BER variation with gaussian noise and ISI

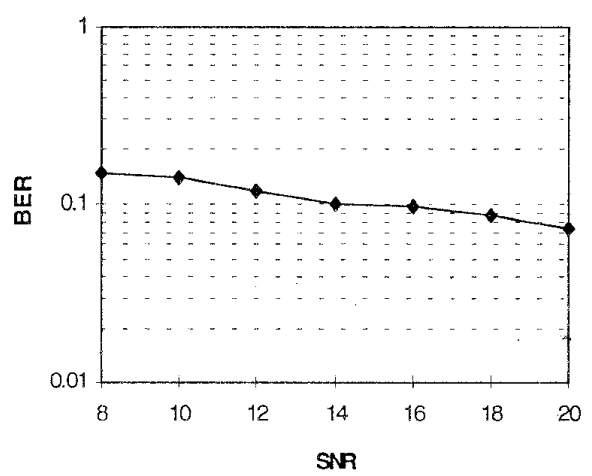

Fig. 5 - BER variation in rural area. 
The limits of this approach are given by the need of switching on different weight matrices depending on the area variations.

It is currently under investigation an unsupervised version of the same recurrent network that allows the real time learning avoiding the problem of several dedicated training phases.

\section{REFERENCES}

[1] Steele R., "Mobile Radio Communications, Pentech Press, 1992.

[2] Qureshi S.U.H., "Adaptive Equalization," Proceedings IEEE, vol.73, No. 9, pp. 1349-1387, 1985.

[3] Pineda F., Dynamics and Architecture for Neural Computation, Journal of Complexity 4, 216-245, Academic Press 1988

[4] Grossberg S., Adaptive Pattern Classification and Universal Recoding: Parallel Development and Coding of Neural Feature Detectors, Biol. Cyber. 23, 121-134, 1976.

[5] Nerrand O., Roussel-Ragot P.,Personnaz L.,Dreyîus G., Neural Networks and Nonlinear Adaptive Filtering: Unifying Concepts and New Algorithms, Neural Computation 5, 165-199, MIT 1992

[6] Williams R.J., Zipser D., A learning Algorithm for Continually Running Fully Recurrent Ncural Networks, Neural Computation 1(2), 270-280, 1989.

[7] Maren A.J., Neural Networks for Spatio-temporal Pattern Recognition, in: Handbook of Neural Computation Applications, ed. by Maren A.J. Harston C.T., Pap R.M., Academic Press 1991.

[8] Rumelhart D.E., Hinton G.E., Williams R.S., ÒLearning Internal Representation by crror Propagation, in : Rumelhart, D.E., McClelland J.L ed.., Parallel Distributed Processing, 318-362, MIT Press, Cambridge 1986.

[9] Pineda F., Generalization of Backpropagation to Recurrent and Higher order Neural Networksî, Physical Review Letters, 18, 2229-2232, 1987.

$[10]$ Proakis J.G., "Digital Communications,"McGrawHill 1983.

[11] Powell M.J.D., Radial Basis Function for Multivariate Interpolation: A Review, Technical Report 1985/NA12 Dept. App. Math and Theor. Phys, Cambridge Un. England 1985.

[12] Maren A., Jones D.,Configuring and Optimizing the Back-Propagation Network, in: Handbook of Neural Computing Applications, ed. by Maren A.J., Harston C.T., Pap R.M., Academic Press 1991

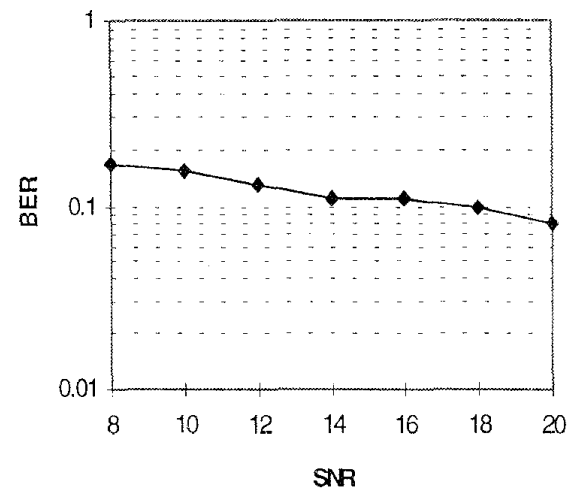

Fig. 6 - BER variation in hilly area

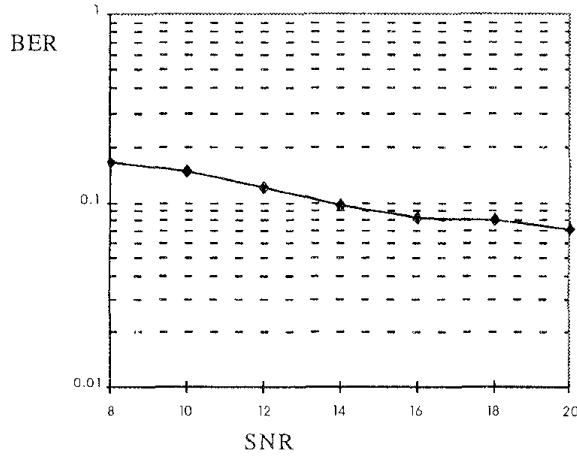

Fig. 7 - BER variation in urban area 